hep-ph/9903395

\title{
Electroweak Symmetry Breaking due to Confinement
}

\author{
Chun Liu \\ Korea Institute for Advanced Study \\ 207-43 Cheongryangri-dong, Dongdaemun-gu, Seoul 130-012, Korea \\ liuc@kias.re.kr
}

\begin{abstract}
Within the framework of gauge mediated supersymmetry breaking, we consider an electroweak symmetry breaking pattern in which there is no conventional $\mu$ term. The pattern is made appealing through realizing it as low energy effective description of a supersymmetric Yang-Mills theory which is of confinement. Phenomenological implications are discussed.
\end{abstract}

PACS numbers: 11.15.Ex, 12.15.-y, 12.60.Fr. 
Supersymmetry [1] provides a solution to the gauge hierarchy problem if it breaks dynamically [2]. It has been realized that the breaking should occur in a hidden sector, which is then communicated to the observable sector. In this paper, we consider the scenario in which the communication is via gauge interactions [3, 4]. Generally, the gauge mediated supersymmetry breaking (GMSB) models have a so-called $\mu$ problem [4, 5], namely either the $\mu$ term is at the weak scale and the $B \mu$ term is unnaturally large, or $B \mu$ is at the weak scale and $\mu$ is very small. That means, there are some difficulties in getting right electro-weak symmetry breaking (EWSB). Although several ways were suggested for solving this problem [4], it would be desirable to find more simple solution.

Instead of generating the $\mu$ term, we suggest to study the EWSB by the following superpotential,

$$
W_{E W S B}=-\lambda X\left(H_{u} H_{d}-\mu^{2}\right)
$$

where $H_{u}$ and $H_{d}$ are the two Higgs doublets, $X$ a standard model singlet; $\mu$ is the EWSB scale, and $\lambda$ the coupling constant. The physical implications of above superpotential will be discussed later. In fact, it was used in early stage of the supersymmetry phenomenology [7]. Spontaneous EWSB is obtained as

$$
v_{u}=v_{d}=\mu
$$

where $v_{u}$ and $v_{d}$ denote vacuum expectation values (vevs) of the doublet Higgs fields, and the other fields have vanishing vevs. Note that $W_{E W S B}$ does not break supersymmetry. After taking relevant soft masses into consideration, it can be seen that there are no light Higgs and light Higgsino particles.

The superpotential $W_{E W S B}$ of Eq. (1) may have fundamental reasons. It can be an effective theory of a more fundamental theory. The results of supersymmetric Yang-Mills theory [8] can be used to realize this idea. To be specific, we exploit a model of Intriligator, Seiberg and Shenker [9]. Introduce a supersymmetric SU(2) gauge interaction with a single matter superfield $Q$ in the $I=3 / 2$ representation. This theory is believed to be of confinement. The basic gauge singlet field is $u=Q^{4}$ with a totally symmetric contraction of the 
gauge indices. The quantum theory has a moduli space of degenerate vacua labeled by the vev of $u$. The nontrivial check of the 't Hooft anomaly matching conditions implies that the Kähler potential at low energy is

$$
K \sim u^{\dagger} u|\Lambda|^{-6} \text { for } \quad u^{\dagger} u<\Lambda^{8}
$$

with $\Lambda$ being the dynamical scale of the $\mathrm{SU}(2)$ interaction. Perturbing the theory by a tree level superpotential $\frac{k}{m} u$ with $m$ being some new physics scale and $k$ the dimensionless coupling coefficient would break supersymmetry. To achieve EWSB other than supersymmetry breaking, we assume that the new physics further couples $Q$ with standard model Higgs fields which are singlet under this $\mathrm{SU}(2)$. The low energy effective superpotential is written as

$$
W_{e f f}=\lambda_{1} m H_{u} H_{d}+\frac{k}{m} u-\frac{c}{m^{3}} u H_{u} H_{d}
$$

where $\lambda_{1}$ and $c$ are dimensionless coupling constants. By field redefinition $u \rightarrow u+m^{4} \frac{\lambda_{1}}{c}$, $W_{\text {eff }}$ becomes to

$$
W_{e f f}=\frac{k}{m} u-\frac{c}{m^{3}} u H_{u} H_{d}
$$

plus some unphysical constant, where we denote the redefined field still as $u$ without confusing. Note that the Kähler potential does not change under this redefinition. To the order of $1 / m^{3}$, the general effective superpotential includes terms $\lambda_{2}\left(H_{u} H_{d}\right)^{2} / m+\lambda_{3}\left(H_{u} H_{d}\right)^{3} / m^{3}$ with $\lambda_{2}$ and $\lambda_{3}$ being dimensionless constants. The presence of these terms does not modify the above-discussed EWSB qualitatively. The point is that whenever there appears a term proportional to $H_{u} H_{d}$, it can be removed through the above procedure of field redefinition. In addition, $\lambda_{2}$ and $\lambda_{3}$ can be small. The smallness is natural in the sense of 't Hooft due to the non-renormalization theorem in supersymmetry. Besides that $u$ is a composite field with dimension 4, Eqs. (3) and (5) is the same as the physics by Eq. (1) with an elementary $X$.

Quantitatively, rescaled field $u / \Lambda^{3}$ corresponds to $X$, and then 


$$
\lambda=c \frac{\Lambda^{3}}{m^{3}}, \quad \mu^{2}=\frac{k}{c} m^{2} .
$$

It can be seen from Eq. (11) that keeping Higgsino mass at weak scale requires $\lambda \sim O(1)$. So numerically $c$ is $\sim m^{3} / \Lambda^{3}>1$. This is consistent with $\mu^{2}<m^{2}$ if $k$ is $O(1), \mu^{2} \sim k \frac{\Lambda}{m} \Lambda^{2}$. By taking $\Lambda$ to be $(100-1000) \mathrm{GeV}, m$ should be about $\left(10^{2}-10^{5}\right) \mathrm{GeV}$. Therefore, viable EWSB can indeed occur dynamically due to confinement of a supersymmetric gauge theory with certain effective tree level superpotential.

It is necessary to discuss theoretical implications of the above described EWSB. First, the breaking scale $\mu$ is not generated by the supersymmetry breaking which has not been dealt with yet. It is related to the $\mathrm{SU}(2)$ dynamical scale $\Lambda$ and the new physics scale $m$. However, the EWSB is still tied to supersymmetry itself. Supersymmetry is necessary to keep the gauge hierarchy whenever there are elementary scalar particles. Second, it is not radiative breaking. Once new scales are introduced for generating the scale $\mu$, radiative breaking is no longer a requirement of simplicity. It is natural to relate the scales $\Lambda$ and $m$ to the EWSB directly. Third, we wonder if there is a relation between the scale $m$ and the supersymmetry breaking scale. For instance, the scale $m$ can be at $10^{4} \mathrm{GeV}$ which might also be the supersymmetry breaking scale. It would be interesting that the EWSB is finally connected to the supersymmetry breaking. Fourth, in principle this EWSB mechanism may also apply to the case of supergravity. Of course, it seems to have less relation with the supersymmetry breaking in this case which is therefore less interesting.

Supersymmetry breaks dynamically in another sector. There are several ways to get the breaking [10]. For simplicity, we can still adopt the model of Ref. [9]. Introduce another $\mathrm{SU}(2)$ with single matter $Q^{\prime}$ in the $I=3 / 2$ representation. The singlet composite field is $u^{\prime}=Q^{\prime 4}$. The tree level superpotential $\frac{u^{\prime}}{m^{\prime}}$ with $m^{\prime}$ being some scale breaks supersymmetry dynamically. To mediate the supersymmetry breaking to the standard model sector, we introduce the so-called messenger fields which are singlet under this new $\mathrm{SU}(2)$ but in the vector representation under the standard model gauge group. Couple $u^{\prime}$ to the messengers in the way like the $u$ field to the Higgs fields in Eq. (4). The difference here is that the 
messenger mass terms in the superpotential cannot be removed by field redefinition so as to avoid the messengers developing vevs. And these mass terms break R-symmetry explicitly [3]. Supersymmetry breaking is mediated to the standard model sector through loops. In fact, the effective theory obtained from the above is just the O'Raifeartaigh model [11] used by Dine and Fischler in Ref. [3] which gives details of the messenger content.

The phenomenological implications of the EWSB described in this paper should be stressed. The Higgs vevs are determined by the superpotential Eq. (11), the supersymmetric standard model gauge interactions and the soft masses,

$$
V=\left|\lambda\left(v_{u} v_{d}-\mu^{2}\right)\right|^{2}+\frac{1}{8}\left(g^{2}+g^{\prime 2}\right)\left(v_{u}^{2}-v_{d}^{2}\right)^{2}+M^{2} v_{u}^{2}+M^{2} v_{d}^{2}
$$

where $g$ and $g^{\prime}$ are the standard model $\mathrm{SU}(2) \times \mathrm{U}(1)$ gauge coupling constants, $M$ the soft mass of the Higgs particles. The minimum of $V$ results in Eq. (2) . Hence

$$
\tan \beta \equiv \frac{v_{u}}{v_{d}}=1
$$

Note that the usual phenomenological constraints on $\tan \beta$ in the minimal supersymmetric standard model (MSSM) do not apply here, because the EWSB is not radiative breaking. Compared with the particle spectra of the MSSM, there is one more neutral Higgs and one more neutralino because of the introduction of $X$ field. Due to the tree level electroweak breaking and the additional coupling $\lambda$, the spectra of the scalar bosons and the neutralinos are less constrained. Nevertheless they are all around the weak scale. Let us look at the neutralino masses which are given as

$$
\left(\begin{array}{lllll}
\tilde{\phi}_{d}^{0} & \tilde{\phi}_{u}^{0} & \tilde{W}^{3} & \tilde{B} & \tilde{X}
\end{array}\right)\left(\begin{array}{ccccc}
0 & 0 & g v_{d} / \sqrt{2} & -g^{\prime} v_{d} / \sqrt{2} & \lambda v_{u} \\
0 & 0 & -g v_{u} / \sqrt{2} & g^{\prime} v_{u} / \sqrt{2} & \lambda v_{d} \\
g v_{d} / \sqrt{2} & -g v_{u} / \sqrt{2} & M_{\tilde{W}} & 0 & 0 \\
-g^{\prime} v_{d} / \sqrt{2} & g^{\prime} v_{u} / \sqrt{2} & 0 & M_{\tilde{B}} & 0 \\
\lambda v_{u} & \lambda v_{d} & 0 & 0 & 0
\end{array}\right),\left(\begin{array}{c}
\tilde{\phi}_{d}^{0} \\
\tilde{\phi}_{u}^{0} \\
\tilde{W}^{3} \\
\tilde{B} \\
\tilde{X}
\end{array}\right),
$$

where $\tilde{\phi}_{d}, \tilde{\phi}_{u}$ and $\tilde{X}$ stand for the fermion components of $H_{d}, H_{u}$ and $X . \tilde{W}$ and $\tilde{B}$ are Wino and Bino with soft masses $M_{\tilde{W}}$ and $M_{\tilde{B}}$ respectively. The determinant of the matrix 
is about $M_{Z}^{4} M_{\tilde{W}}$. We see explicitly that there is no light Higgsino. And all the neutralinos are around the weak scale. The chargino mass matrix is more predictive,

$$
\left(\begin{array}{cc}
\tilde{\phi}_{u}^{+} & \tilde{W}^{+}
\end{array}\right)\left(\begin{array}{cc}
0 & M_{W} \\
M_{W} & M_{\tilde{W}}
\end{array}\right)\left(\begin{array}{c}
\tilde{\phi}_{u}^{-} \\
\tilde{W}^{-}
\end{array}\right) .
$$

Because of the absence of conventional $\mu$ term, the two chargino mass product satisfies: $M_{\tilde{\chi}_{1}^{ \pm}} M_{\tilde{\chi}_{2}^{ \pm}}=M_{W}^{2} \cdot M_{\tilde{W}} \neq 0$ leads to that one of the charginos must be lighter than the $\mathrm{W}$ boson. Such a chargino is within the experimental reach. However, if the lightest neutralino mass is close to this chargino mass within a few $\mathrm{GeV}$, it is hard to be detected.

In summary, an old EWSB pattern has been re-suggested to avoid the $\mu$ problem in the GMSB scenario. Our main point is that it can be effective description of a more fundamental supersymmetric gauge theory which is of confinement. Phenomenologically, additional neutral Higgs and one more neutralino are predicted with masses around the weak scale. One of the charginos is lighter than the $\mathrm{W}$ gauge boson.

Several remarks should be made finally. (i) This model is originally motivated by the works of Ref. [12] which aim at the flavor problem. Slight lepton number violation can be introduced into the model. The scalar neutrinos develop small vevs. In this case the $\tan \beta$ deviates from unity slightly. (ii) The relation between EWSB and super-Yang-Mills theory is not unique. In a recent model of Ref. [13], conventional $\mu$ term is generated dynamically. (iii) This EWSB mechanism is similar to the spirit of the technicolor [14]. The electroweak symmetry breaking is triggered by a strong $\mathrm{SU}(2)$ interaction. However, there is distinction, that is the property of the strong gauge interaction used in this mechanism is not spontaneous chiral symmetry breaking, but confinement.

\section{ACKNOWLEDGMENTS}

I would like to thank E.J. Chun and S.Y. Choi for helpful discussions. 


\section{REFERENCES}

[1] J. Wess and B. Zumino, Nucl. Phys. B70, 39 (1974);

Y. Gol'fand and E. Likhtam, JETP Lett. 13, 323 (1971);

D.V. Volkov and V. Akulov, Phys. Lett. B46, 109 (1973).

[2] E. Witten, Nucl. Phys. B188, 513 (1981).

[3] M. Dine and W. Fischler, Phys. Lett. B110, 227 (1982);

L. Alvarez-Gaumé, M. Claudson and M. Wise, Nucl. Phys. B207, 96 (1982);

C.R. Nappi and B.A. Ovrut, Phys. Lett. B113, 175 (1982).

[4] M. Dine and A.E. Nelson, Phys. Rev. D48, 1277 (1993);

M. Dine, A.E. Nelson and Y. Shirman, Phys. Rev. D51, 1362 (1995);

M. Dine, A.E. Nelson, Y. Nir and Y. Shirman, Phys. Rev. D53, 2658 (1996).

[5] G. Dvali, G.F. Giudice and A. Pomarol, Nucl. Phys. B478, 31 (1996).

[6] S. Dimopoulos, G. Dvali and R. Rattazi, Phys. Lett. B413, 336 (1997); T. Yanagida, Phys. Lett. B400, 109 (1997); P. Ciafaloni and A. Pomarol, Phys. Lett. B404, 83 (1997); H.P. Nilles and N. Polonsky, Phys. Lett. B412, 69 (1997); G. Dvali and M. Shifman, Phys. Lett. B399, 60 (1997); A. de Gouvêa, A. Friedland and H. Murayama, Phys. Rev. D57, 5676 (1998); E.J. Chun, Phys. Rev. D59, 015011 (1999).

[7] For reviews, see H.P. Nilles, Phys. Rep. 110, 1 (1984);

R.N. Mohapatra, Unification and Supersymmetry (Springer-Verlag, 1986).

[8] N. Seiberg, Phys. Lett. B318, 469 (1993); Phys. Rev. D49, 6857 (1994); Nucl. Phys. B435, 129 (1995).

[9] K. Intriligator, N. Seiberg and S.H. Shenker, Phys. Lett. B342, 152 (1995).

[10] For reviews, see W. Skiba, Mod. Phys. Lett. A12, 737 (1997);

E. Poppitz and S.P. Trivedi, hep-th/9803107; 
L. Randall, in Nagoya 1996 (Perspectives of Strong Coupling Gauge Theories), p.258;

S. Thomas, in Nagoya 1996 (Perspectives of Strong Coupling Gauge Theories), p.272.

[11] L. O'Raifeartaigh, Nucl. Phys. B96, 331 (1975).

[12] D. Du and C. Liu, Mod. Phys. Lett. A8, 2271 (1993); A10, 1837 (1995);

C. Liu, Mod. Phys. Lett. A12, 329 (1997); Int. J. Mod. Phys. A11, 4307 (1996);

C. Liu and H.S. Song, Nucl. Phys. B545, 183 (1999), hep-ph/9811203.

[13] K. Choi, H.B. Kim and H.D. Kim, talk at KIAS Winter School on B and CP violation (Feb. 22-28, 1999).

[14] L. Susskind, Phys. Rev. D20, 2619 (1979);

S. Weinberg, Phys. Rev. D19, 1277 (1978). 